\title{
Fully implicational methods for interval-valued fuzzy reasoning with multi-antecedent rules
}

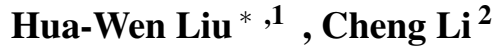 \\ ${ }^{1}$ School of Mathematics, Shandong University, \\ Jinan, Shandong 250100, China \\ E-mail:hw.liu@sdu.edu.cn \\ ${ }^{2}$ School of Mathematics, Shandong University, \\ Jinan, Shandong 250100, China \\ E-mail: licheng19861120@hotmail.com \\ Received 1 September 2011 \\ Accepted 18 September 2011
}

\begin{abstract}
Based on the fully implicational idea, we investigate the interval-valued fuzzy reasoning with multiantecedent rules. First, we construct a class of interval-valued fuzzy implications by means of a type of implications and a parameter on the unit interval, then use them to establish three kinds of fully implicational reasoning methods for the interval-valued fuzzy reasoning with multi-antecedent rules which are called triple I method, hierarchical triple I method and URC triple I method respectively. At the same time, we analyze the Modus-Ponens (MP for short) and continuity properties of these methods and investigate the equivalence between the first method and the latter two methods. We also restrict our discussion to Zadeh fuzzy set theory and obtain a series of corresponding results.
\end{abstract}

Keywords: Approximate reasoning; interval-valued fuzzy sets; interval-valued fuzzy implications; fully implicational methods; Modus-Ponens property; fuzzy implications.

\section{Introduction}

The compositional rule of inference (CRI) proposed by Zadeh [1] is one of the earliest and most important inference scheme in fuzzy reasoning. By the CRI, for a given fuzzy rule "if $u$ is $A$ then $v$ is $B$ " (briefly $A \Rightarrow B$ ) and a fact " $u$ is $A^{\prime \prime}$, the inferred output $B^{\prime}$ is obtained by the following

$$
B^{\prime}(v)=\sup _{u \in U} T\left(A^{\prime}(u), R(A(u), B(v))\right), v \in V
$$

where $T$ is a t-norm, $R$ is a fuzzy relation, and $A, A^{\prime}, B$ and $B^{\prime}$ are fuzzy sets on their respective do-

\footnotetext{
* Corresponding author.
}

mains $U$ and $V$. To improve the CRI, Wang [2] proposed a new reasoning method called fully implicational triple I (the abbreviation of triple implications) method (shortly, triple I method) only using implications on $[0,1]$. This new method has been extensively discussed later (see, e.g.[2-17]) and its principle followed is as follows: for given $\alpha \in[0,1]$, rule $A \Rightarrow B$ and fact $A^{\prime}$, the inferred $B^{\prime}$ should be the smallest fuzzy subset of $V$ satisfying

$$
(A(u) \rightarrow B(v)) \rightarrow\left(A^{\prime}(u) \rightarrow B^{\prime}(v)\right) \geqslant \alpha
$$

for all $u \in U$ and $v \in V$, where $\rightarrow$ denotes an implication $I$ on $[0,1]$. Inequality 2 indicates that the 
sustaining degree [2] of $A \Rightarrow B$ to $A^{\prime} \Rightarrow B^{\prime}$ should be greater than or equal to $\alpha$. The above principle is called "the $\alpha$-triple I principle". When $\alpha=1$, then we have "the triple I principle". In [6], we established the following computing formula for $B^{\prime}$ according to the triple I principle:

$$
B^{\prime}(v)=\sup _{u \in U} T\left(T(\alpha, I(A(u), B(v))), A^{\prime}(u)\right), \quad v \in V
$$

where $I$ is an implication on $[0,1]$ satisfying (P5) and (P) and the mapping $T$ is its residual (see Propositions 1 and 2 in this paper). Especially, if $\alpha=1$ and the mapping $T$ satisfies $T(1, b)=b$ for any $b \in[0,1]$ in (3), then we have

$$
B^{\prime}(v)=\sup _{u \in U} T\left(I(A(u), B(v)), A^{\prime}(u)\right), v \in V
$$

It is clear that (4) is identical to (1) when $T$ is a tnorm. From this point of view, we can say that the triple I method improves the CRI.

Interval-valued fuzzy sets (IVFSs for short) [18, 19] are extensions of Zadeh's fuzzy sets [20]. Owing to the effective and reasonable description to the uncertainty information, the expression ability of IVFSs is stronger than that of Zadeh's fuzzy sets. So, it is hopeful to improve the technology of approximate reasoning by using IVFSs. Türksen et al. [19, 21, 22], Gorzałczany et al. [23-26], Bustince [27] and $\mathrm{Wu}$ [28] et al. all studied the approximate reasoning with IVFSs. The methods used by them are based on CRI or similarity measures. Based on fully implicational idea, Wang et al. [11, 29] also studied the interval-valued fuzzy reasoning (briefly IVFR). But the inference model differs from the ordinary one. Wang [12] handled IVFR by means of the triple I method, but the employed interval-valued fuzzy t-norms were only t-representable in the sense of Deschrijver et al.[30]. The approach linked to these t-norms is the simplest and most intuitive one. As shown in [31], these t-norms reveal only a small tip of the iceberg while much more remains hidden beneath the surface. In the present paper, we will apply the fully implicational idea to the IVFR. At first, motivated by the form of a class of interval-valued fuzzy implications constructed by Deschrijver and Kerre [32, 33], which possess more interesting and applicable properties as shown in [31], we will construct a class of interval-valued fuzzy implications using a type of implications on [0,1] only satisfying (P5) and (P) (see Proposition 1 in this paper), then use them to establish the fully implicational methods for the reasoning with multi-antecedent rules.

Recently, aiming at the reasoning with multiantecedent rules, Balasubramaniam [34] proposed a novel modified scheme of classical CRI inferencing called hierarchical CRI which has some advantages over the classical one, and also showed that the hierarchical CRI and the classical CRI are equivalent under some conditions. Comds and Andrews [35] presented a rule configuration called union rule configuration (URC, for short) for the multi-antecedent rule. The URC can eliminate the combinatorial explosion of rules as inputs are added to the system. As shown in [35] and [34], the above hierarchical CRI and URC have many advantages over the classical ones. So, in the present paper, we will also apply the fully implicational idea to the hierarchical and URC methods, and propose hierarchical and URC fullimplicational methods. Moreover, the MP property of these methods and the equivalence between the classical and the two new full-implicational methods will be investigated. For the case of Zadeh fuzzy sets, a series of corresponding results will be obtained.

The outline of this paper is as follows. In the next section, we briefly recall some preliminaries and construct a class of interval-valued fuzzy implications. In Section 3, we establish the fully implicational method for the IVFR with multi-antecedent rules and investigate its MP and continuity properties. Sections 4 and 5 propose respectively the hierarchical and URC full-implicational methods for the IVFR with multi-antecedent rules and discuss their MP continuity properties. The equivalence between the classical full-implicational method and the hierarchical and URC full-implicational methods is also investigated. The works in Sections 3, 4, and 5 also include the case of Zadeh fuzzy sets. The final section is the conclusion. 


\section{Interval-valued fuzzy implications}

Firstly, to make this paper self-contained, we briefly recall some of the concepts and results employed in the rest of this paper. Then, a class of intervalvalued fuzzy implications are constructed by means of a type of implications and a parameter on $[0,1]$.

Definition 1. ([36-39]) An implication on $[0,1]$ is any $[0,1]^{2} \rightarrow[0,1]$ mapping $I$ satisfying $I(0,0)=$ $I(0,1)=I(1,1)=1$ and $I(1,0)=0$. We also require that $I$ is nonincreasing in its first and nondecreasing in its second component. The implication generated by a t-norm $T$ is defined as

$$
I_{T}(x, y)=\sup \{\gamma \in[0,1] \mid T(x, \gamma) \leqslant y\}, \quad x, y \in[0,1]
$$

It follows from the definition above that $I(0, a)=$ 1 and $I(a, 1)=1$ for all $a \in[0,1]$.

Definition 2. Let $T$ and $I$ be two $[0,1]^{2} \rightarrow[0,1]$ mappings, $T$ and $I$ are said to be residual to each other, if the following residuation condition holds for all $x, y, z \in[0,1]$,

$$
T(x, y) \leqslant z \text { if and only if } x \leqslant I(y, z)
$$

Proposition 1. ([13, 40-42]) Let $T$ be a leftcontinuous t-norm on $[0,1]$, then its residuum $I_{T}$ (written by I for short) defined by (5) has the following properties:

$$
\begin{aligned}
& \text { (P1) } I(y, z)=1 \Longleftrightarrow y \leqslant z ; \\
& (P 2) x \leqslant I(y, z) \Longleftrightarrow y \leqslant I(x, z) ; \\
& (P 3) I(x, I(y, z))=I(y, I(x, z)) ; \\
& (P 4) I(1, z)=z ; \\
& \text { (P5) } I\left(x, \inf _{z \in Z} z\right)=\inf _{z \in Z} I(x, z) ; \\
& \text { (P6) } I\left(\sup _{z \in Z} z, y\right)=\inf _{z \in Z} I(z, y),
\end{aligned}
$$

where $x, y, z \in[0,1]$ and $Z$ is any nonempty subset of $[0,1]$.

Proposition 2. ([6]) If a mapping $I:[0,1]^{2} \rightarrow[0,1]$ satisfies (P5) and

(P) $\{\gamma \in[0,1] \mid x \leqslant I(y, \gamma)\} \neq \emptyset$ for all $x, y \in[0,1]$, then the mapping $T:[0,1]^{2} \rightarrow[0,1]$ defined by

$$
T(x, y)=\inf \{\gamma \in[0,1] \mid x \leqslant I(y, \gamma)\}, \quad x, y \in[0,1]
$$

is residual to I, i.e., $T$ and I satisfy the residuation condition (6).

Interval-valued fuzzy sets, which were apparently first studied by Sambuc [18], are the extensions of Zadeh's fuzzy sets [20] and their definition is as follows.

Definition 3. An interval-valued fuzzy set (IVFS, for short) on a universe $U$ is a mapping $A: U \rightarrow L^{I}$, $A(u)=\left[\mu_{A}(u), v_{A}(u)\right] \in L^{I}$ for any $u \in U$, where $L^{I}=\left\{\left[x_{1}, x_{2}\right] \mid x_{1}, x_{2} \in[0,1]\right.$ and $\left.x_{1} \leqslant x_{2}\right\}$. The order $\leqslant_{L^{I}}$ on $L^{I}$ is defined by $\left[x_{1}, x_{2}\right] \leqslant_{L^{I}}\left[y_{1}, y_{2}\right] \Longleftrightarrow x_{1} \leqslant$ $y_{1}$ and $x_{2} \leqslant y_{2}$. We also write $\left[x_{1}, x_{2}\right] \leqslant L^{I}\left[y_{1}, y_{2}\right]$ as $\left[y_{1}, y_{2}\right] \geqslant_{L^{I}}\left[x_{1}, x_{2}\right]$. We denote the set of all IVFSs on $U$ by $\operatorname{IVFS}(\mathrm{U})$.

The lattice $\left(L^{I}, \leqslant_{L^{I}}\right)$ is a complete lattice [43] and its smallest and greatest elements are denoted as $0_{L^{I}}=[0,0]$ and $1_{L^{I}}=[1,1]$, respectively. An IVFS $A$ can be seen as an L-fuzzy set in the sense of Goguen [44] with respect to the lattice $L^{I}$. The operators sup and inf on $L^{I}$ are defined as follows, for any nonempty subset $Z$ of $L^{I}$,

$$
\begin{aligned}
& \sup \{z \mid z \in Z\}=\left[\sup \left\{z_{1} \mid z \in Z\right\}, \sup \left\{z_{2} \mid z \in Z\right\}\right], \\
& \inf \{z \mid z \in Z\}=\left[\inf \left\{z_{1} \mid z \in Z\right\}, \inf \left\{z_{2} \mid z \in Z\right\}\right] .
\end{aligned}
$$

From now on, if $x \in L^{I}$ then the left end point of $x$ is denoted by $x_{1}$ or $\operatorname{Pr}_{1} x$ and the right end point by $x_{2}$ or $\operatorname{Pr}_{2} x$, i.e. $x=\left[x_{1}, x_{2}\right]$ or $x=\left[\operatorname{Pr}_{1} x, \operatorname{Pr}_{2} x\right]$. If $x_{1}=x_{2}$, then $\left[x_{1}, x_{2}\right]$ can also be written as $x_{1}$ or $x_{2}$ simply.

Cornelis, Deschrijver and Kerre [30, 33, 45] generalized the definitions of t-norms and implications on $[0,1]$ to $L^{I}$ as follows.

Definition 4. A triangular norm (briefly $t$-norm) on $L^{I}$ (also called interval-valued fuzzy t-norm) is any increasing, commutative, associative $\left(L^{I}\right)^{2} \rightarrow L^{I}$ mapping $\mathscr{T}$ satisfying $\mathscr{T}\left(1_{L^{I}}, x\right)=x$ for all $x \in L^{I}$.

Definition 5. An implication on $L^{I}$ (also called interval-valued fuzzy implication) is any $\left(L^{I}\right)^{2} \rightarrow L^{I}$ mapping $\mathscr{I}$ which is nonincreasing in its first and nondecreasing in its second component, and which satisfies $\mathscr{I}\left(0_{L^{I}}, 0_{L^{I}}\right)=\mathscr{I}\left(0_{L^{I}}, 1_{L^{I}}\right)=\mathscr{I}\left(1_{L^{I}}, 1_{L^{I}}\right)=$ $1_{L^{I}}$ and $\mathscr{I}\left(1_{L^{I}}, 0_{L^{I}}\right)=0_{L^{I}}$. The implication generated by a t-norm $\mathscr{T}$ on $L^{I}$ is defined for all $x, y \in L^{I}$ as

$$
\mathscr{I}_{\mathscr{T}}(x, y)=\sup \left\{\gamma \in L^{I} \mid \mathscr{T}(x, \gamma) \leqslant L^{I} y\right\}
$$


We also write $\mathscr{I}(x, y)$ as $x \rightarrow y$ in our discussion.

Definition 6. ([31]) Given a t-norm $T$ on [0,1], the $\left(L^{I}\right)^{2} \rightarrow L^{I}$ mapping $\mathscr{T}_{T}^{p}$ defined by, for $x, y$ in $L^{I}$,

$$
\mathscr{T}_{T}^{p}(x, y)=\left[T\left(x_{1}, y_{1}\right), \max \left\{T\left(x_{1}, y_{2}\right), T\left(x_{2}, y_{1}\right)\right\}\right]
$$

is a t-norm on $L^{I}$ that is called the pessimistic t-norm with representative $T$.

Deschrijver and Kerre constructed in [33, 46] a class of R-implications on $L^{I}$ by means of the residua generated by left-continuous t-norms on $[0,1]$, which is shown in [31] as a class of residual implications generated by the generalized pessimistic t-norms on $L^{I}$ (see [31]). Deschrijver et al. [31] also pointed out that from the point of view of induced properties the most interesting connectives are the pessimistic t-norms together with their (optimistic) residual implications. This means that this class of R-implications on $L^{I}$ will have good application perspectives. So, we consider to employ this class of implications in our work. In our study on reasoning methods, however, we find that this generating condition is very strong, and as a result, some common implications, such as Kleene-Dienes implication, Reichenbach implication, Yager implication and so on, can not be used to establish the reasoning formulas. So, in the present paper, we firstly generalize Deschrijver and Kerre's interval-valued fuzzy implications to a new form by using the implications on $[0,1]$ only satisfying $(\mathrm{P} 5)$ and $(\mathrm{P})$, then, use them to establish the computing formulas for fuzzy reasoning with multi-antecedent rules.

Theorem 3. Suppose that I is an implication on $[0,1]$ satisfying $(P 5)$ and $(P)$, mapping $T$ defined by (7) is its residual, and $t \in[0,1]$. Then the mapping $\mathscr{I}_{I, t}:\left(L^{I}\right)^{2} \rightarrow L^{I}$ defined by

$$
\begin{aligned}
\mathscr{I}_{I, t}(x, y)= & {\left[\min \left\{I\left(x_{1}, y_{1}\right), I\left(x_{2}, y_{2}\right)\right\},\right.} \\
& \left.\min \left\{I\left(T\left(x_{2}, t\right), y_{2}\right), I\left(x_{1}, y_{2}\right)\right\}\right]
\end{aligned}
$$

for all $x, y \in L^{I}$, is an implication on $L^{I}$.

Proof. Since $T(0, a)=\inf \{c \in[0,1] \mid 0 \leqslant I(a, c)\}=$ 0 for all $a \in[0,1]$ and $I$ is an implication on $[0,1]$, it is very easy to verify that $\mathscr{I}_{I, t}\left(0_{L^{I}}, 0_{L^{I}}\right)=\mathscr{I}_{I, t}\left(0_{L^{I}}, 1_{L^{I}}\right)=\mathscr{I}_{I, t}\left(1_{L^{I}}, 1_{L^{I}}\right)=1_{L^{I}}$ and $\mathscr{I}_{I, t}\left(1_{L^{I}}, 0_{L^{I}}\right)=0_{L^{I}}$ hold. Formula (7) follows that $T$ is nondecreasing in its first component. So, from the mixed monotonicity of the implication $I$, we also easily verify that $\mathscr{I}_{I, t}$ is nonincreasing in its first and nondecreasing in its second component.

Corollary 4. Under the same assumptions of Theorem 3, if $t=0$, then the implication defined by (8) is just the optimistic implication on $L^{I}$ as one of the pseudo-i-representable implication [46], for $x, y \in L^{I}$,

$$
\mathscr{I}_{I}^{o}(x, y)=\left[\min \left\{I\left(x_{1}, y_{1}\right), I\left(x_{2}, y_{2}\right)\right\}, I\left(x_{1}, y_{2}\right)\right]
$$

Proof. It follows from $I(0,0)=1$ that $0 \in\{c \in$ $[0,1] \mid a \leqslant I(0, c)\}$. So we have $T(a, 0)=0$ for any $a \in[0,1]$. Again, $I(0, b)=1(\forall b \in[0,1])$ for any implication $I$ on $[0,1]$ in the sense of Definition 1. Therefore, we can easily get (9) from (8) when $t=0$.

We now generalize Definition 2 to the case of $L^{I}$.

Definition 7. Let $\mathscr{T}$ and $\mathscr{I}$ be two $\left(L^{I}\right)^{2} \rightarrow L^{I}$ mappings, $\mathscr{T}$ and $\mathscr{I}$ are said to be residual to each other, if the following residuation condition holds for all $x, y, z \in L^{I}$,

$$
\mathscr{T}(x, y) \leqslant_{L^{I}} z \text { if and only if } x \leqslant_{L^{I}} \mathscr{I}(y, z)
$$

Theorem 5. Let I be an implication on [0,1] satisfying (P5) and $(P)$ and $t \in[0,1]$ be fixed. We define the the mapping $\mathscr{T}:\left(L^{I}\right)^{2} \rightarrow L^{I}$ by

$$
\mathscr{T}(x, y)=\inf \left\{\gamma \in L^{I} \mid x \leqslant_{L^{I}} \mathscr{I}_{I, t}(y, \gamma)\right\}, x, y \in L^{I}
$$

Then $\mathscr{T}$ can be expressed as follows, for any $x, y \in$ $L^{I}$ :

$$
\begin{aligned}
\mathscr{T}(x, y)= & {\left[T\left(x_{1}, y_{1}\right), \max \left\{T\left(x_{2}, T\left(y_{2}, t\right)\right), T\left(x_{1}, y_{2}\right),\right.\right.} \\
& \left.\left.T\left(x_{2}, y_{1}\right)\right\}\right],
\end{aligned}
$$

and it is residual to $\mathscr{I}_{I, t}$ defined by (8), i.e., $\mathscr{T}$ and $\mathscr{I}_{I, t}$ satisfy (10). 
Proof. Since implication I satisfies (P5) and (P), Proposition 2 follows that the mapping $T$ defined by (7) is residual to $I$. For any $x, y, \gamma \in L^{I}$, we have

$$
\begin{array}{ll} 
& x_{1} \leqslant \operatorname{Pr}_{1} \mathscr{I}_{I, t}(y, \gamma) \\
\text { iff } & x_{1} \leqslant \min \left\{I\left(y_{1}, \gamma_{1}\right), I\left(y_{2}, \gamma_{2}\right)\right\} \\
\text { iff } & \left.x_{1} \leqslant I\left(y_{1}, \gamma_{1}\right) \text { and } x_{1} \leqslant I\left(y_{2}, \gamma_{2}\right)\right\} \\
\text { iff } & T\left(x_{1}, y_{1}\right) \leqslant \gamma_{1} \text { and } T\left(x_{1}, y_{2}\right) \leqslant \gamma_{2}, \\
& x_{2} \leqslant \operatorname{Pr}_{2} \mathscr{I}_{I, t}(y, \gamma) \\
\text { iff } \quad & x_{2} \leqslant \min \left\{I\left(T\left(y_{2}, t\right), \gamma_{2}\right), I\left(y_{1}, \gamma_{2}\right)\right\} \\
\text { iff } & \left.x_{2} \leqslant I\left(T\left(y_{2}, t\right), \gamma_{2}\right) \text { and } x_{2} \leqslant I\left(y_{1}, \gamma_{2}\right)\right\} \\
\text { iff } & T\left(x_{2}, T\left(y_{2}, t\right)\right) \leqslant \gamma_{2} \text { and } T\left(x_{2}, y_{1}\right) \leqslant \gamma_{2} .
\end{array}
$$

So we get the expression of $\mathscr{T}$ defined by (11) as follows

$$
\begin{aligned}
\mathscr{T}(x, y)= & \inf \left\{\gamma \in L^{I} \mid x \leqslant_{L^{I}} \mathscr{I}_{I, t}(y, \gamma)\right\} \\
= & {\left[T\left(x_{1}, y_{1}\right), \max \left\{T\left(x_{2}, T\left(y_{2}, t\right)\right),\right.\right.} \\
& \left.\left.T\left(x_{1}, y_{2}\right), T\left(x_{2}, y_{1}\right)\right\}\right] .
\end{aligned}
$$

Further, by the definition of order $\leqslant_{L^{I}}$, Proposition 2 and formula (8), we have for any $x, y, z \in L^{I}$ that

$$
\begin{aligned}
& \mathscr{T}(x, y) \leqslant L^{I} z \\
& \text { iff } \quad T\left(x_{1}, y_{1}\right) \leqslant z_{1} \text { and } \max \left\{T\left(x_{2}, T\left(y_{2}, t\right)\right), T\left(x_{1}, y_{2}\right),\right. \\
& \left.\quad T\left(x_{2}, y_{1}\right)\right\} \leqslant z_{2} \\
& \text { iff } x_{1} \leqslant I\left(y_{1}, z_{1}\right), T\left(x_{2}, T\left(y_{2}, t\right)\right) \leqslant z_{2}, T\left(x_{1}, y_{2}\right) \leqslant z_{2} \\
& \quad \text { and } T\left(x_{2}, y_{1}\right) \leqslant z_{2} \\
& \text { iff } x_{1} \leqslant I\left(y_{1}, z_{1}\right), x_{2} \leqslant I\left(T\left(y_{2}, t\right), z_{2}\right), x_{1} \leqslant I\left(y_{2}, z_{2}\right) \\
& \quad \text { and } x_{2} \leqslant I\left(y_{1}, z_{2}\right) \\
& \text { iff } x_{1} \leqslant \min \left\{I\left(y_{1}, z_{1}\right), I\left(y_{2}, z_{2}\right)\right\} \text { and } \\
& \quad x_{2} \leqslant \min \left\{I\left(T\left(y_{2}, t\right), z_{2}\right), I\left(y_{1}, z_{2}\right)\right\} \\
& \text { iff } x \leqslant L^{I} \mathscr{I}_{I, t}(y, z), \\
& \text { i.e., } \mathscr{T} \text { is residual to } \mathscr{I}_{I, t} .
\end{aligned}
$$

Remark 1. The mapping $\mathscr{T}$ given by (12) is not necessarily a t-norm on $L^{I}$. For instance, the KleeneDienes implication defined by $I(a, b)=(1-a) \vee b$ satisfies (P5) and (P), and its residual mapping is as follows:

$$
T(a, b)=\left\{\begin{array}{l}
0, \text { if } a+b \leqslant 1 \\
a, \text { if } a+b>1
\end{array}, \quad(a, b \in[0,1]),\right.
$$

but the $\mathscr{T}$ given by (12) is not a t-norm on $L^{I}$ because $T(1, b) \neq b$ for all $b \in(0,1)$ and hence $\mathscr{T}\left(1_{L^{I}}, y\right) \neq y$ for any $y \in L^{I} \backslash\left\{0_{L^{I}}, 1_{L^{I}}\right\}$.

Definition 8. An interval-valued fuzzy set $A$ on a nonempty set $U$ is said to be a "singleton" if there exists an $u_{0} \in U$ such that

$$
A(u)=\left\{\begin{array}{ll}
1_{L^{I}}, & \text { if } u=u_{0} \\
0_{L^{I}}, & \text { if } u \neq u_{0}
\end{array} .\right.
$$

We say $A$ attains normality at $u_{0} \in U$. A Zadeh fuzzy set $A$ on the set $U$ is said to be a "singleton" if there exists an $u_{0} \in U$ such that

$$
A(u)=\left\{\begin{array}{ll}
1, & \text { if } u=u_{0} \\
0, & \text { if } u \neq u_{0}
\end{array} .\right.
$$

\section{Fully implicational reasoning method}

A multi-antecedent rule of the form

If $u$ is $A$ and $v$ is $B$, then $w$ is $C$

denoted by $(A, B) \Rightarrow C$ for simplicity, is usually represented by a fuzzy relation $R(u, v, w): U \times V \times W \rightarrow$ $L^{I}$ as follows:

$$
R(u, v, w)=\mathscr{I}(\mathscr{T}(A(u), B(v)), C(w))
$$

where $A, B$ and $C$ are IVFSs on their respective domains $U, V$ and $W$, and $\mathscr{I}$ and $\mathscr{T}$ are respectively implication and t-norm on $L^{I}$. Given a multi-input $\left(A^{\prime}, B^{\prime}\right)\left(A^{\prime}\right.$ and $B^{\prime}$ are IVFSs on $U$ and $V$, respectively) and $\alpha \in L^{I}$, according to the classical triple I method, we can obtain the inferred output $C^{\prime}$ as shown in the following.

Theorem 6. ( $\alpha$-triple I reasoning method) Suppose that the interval-valued fuzzy implication $\mathscr{I}_{I, t}$ defined by (8) is used, where $t \in[0,1]$ and implication I on $[0,1]$ satisfies $(P 5)$ and $(P)$. Then for the given rule (13), input $\left(A^{\prime}, B^{\prime}\right)$ and any arbitrarily fixed $\alpha \in L^{I}$, the output $C^{\prime}$ can be expressed as follows:

$$
\begin{gathered}
C^{\prime}(w)=\sup _{u \in U, v \in V} \mathscr{T}\left(\mathscr { T } \left(\alpha, \mathscr{I}_{I, t}(\mathscr{T}(A(u), B(v)),\right.\right. \\
\left.C(w))), \mathscr{T}\left(A^{\prime}(u), B^{\prime}(v)\right)\right), w \in W
\end{gathered}
$$


where $\mathscr{T}$ is the mapping residual to $\mathscr{I}_{I, t}$.

Proof. According to the triple I principle of fuzzy reasoning, the output $C^{\prime}$ should be the smallest IVFS on $W$ satisfying the following inequality for any $w \in W:$

$$
\begin{aligned}
& \mathscr{I}_{I, t}\left(\mathscr{I}_{I, t}(\mathscr{T}(A(u), B(v)), C(w)), \mathscr{I}_{I, t}\left(\mathscr { T } \left(A^{\prime}(u),\right.\right.\right. \\
& \left.\left.\left.B^{\prime}(v)\right), C^{\prime}(w)\right)\right) \geqslant_{L^{I}} \alpha, \quad u \in U, v \in V
\end{aligned}
$$

Since implication $I$ satisfies (P5) and (P), $\mathscr{I}_{I, t}$ satisfies residuation condition (10). So (16) is equivalent to the following:

$$
\begin{aligned}
& \mathscr{T}\left(\mathscr{T}\left(\alpha, \mathscr{I}_{I, t}(\mathscr{T}(A(u), B(v)), C(w))\right), \mathscr{T}\left(A^{\prime}(u),\right.\right. \\
& \left.\left.B^{\prime}(v)\right)\right) \leqslant_{L^{I}} C^{\prime}(w) .
\end{aligned}
$$

Hence, the smallest IVFS on $W$ satisfying (16) is the $C^{\prime}$ defined by (15).

Remark 2. For the given rule $(A, B) \Rightarrow C$ and the input $\left(A^{\prime}, B^{\prime}\right)$, according to the triple $I$ principle of fuzzy reasoning [13], the fact that $\left(A^{\prime}, B^{\prime}\right)$ implies the output $C^{\prime}$ should be considered and should also be fully sustained by the major premise $(A, B) \Rightarrow C$, i.e.,

$$
\begin{aligned}
& (\mathscr{T}(A(u), B(v)) \rightarrow C(w)) \rightarrow\left(\mathscr{T}\left(A^{\prime}(u), B^{\prime}(v)\right) \rightarrow\right. \\
& \left.C^{\prime}(w)\right)
\end{aligned}
$$

should take its maximum for all $u \in U, v \in V, w \in W$, where $\rightarrow$ denotes an implication $\mathscr{I}$ on $L^{I}$. For a given $\alpha \in L^{I}$, the $\alpha$-triple principle means that the major premise $(A, B) \Rightarrow C$ should sustain the new formed rule $\left(A^{\prime}, B^{\prime}\right) \Rightarrow C^{\prime}$ on the $\alpha$-level, i.e., the following inequality holds for all $u \in U, v \in V, w \in W$ :

$$
\begin{aligned}
& (\mathscr{T}(A(u), B(v)) \rightarrow C(w)) \rightarrow\left(\mathscr{T}\left(A^{\prime}(u), B^{\prime}(v)\right) \rightarrow\right. \\
& \left.C^{\prime}(w)\right) \geqslant_{L^{I}} \alpha .
\end{aligned}
$$

Corollary 7. If the $A, A^{\prime}, B, B^{\prime}$ and $C$ are all Zadeh fuzzy sets on their respective domains $U, V$ and $W$, the employed implication I on [0,1] satisfies (P5) and $(P)$, then the output $C^{\prime}$ can be obtained in a similar way as in Theorem 6:

$$
\begin{gathered}
C^{\prime}(w)=\sup _{u \in U, v \in V} T(T(\alpha, I(T(A(u), B(v)), C(w))), \\
\left.T\left(A^{\prime}(u), B^{\prime}(v)\right)\right), \quad w \in W
\end{gathered}
$$

where $\alpha \in[0,1]$ is any arbitrarily fixed and the mapping $T$ is residual to $I$.

Corollary 8. (Triple I reasoning method) Under the same conditions as Theorem 6, suppose that implication I also satisfies (P1) and (P4), and $\alpha=1_{L^{I}}$, then for given rule (13) and input $\left(A^{\prime}, B^{\prime}\right)$, the output $C^{\prime}$ can be expressed as follows:

$$
\begin{gathered}
C^{\prime}(w)=\sup _{u \in U, v \in V} \mathscr{T}\left(\mathscr{I}_{I, t}(\mathscr{T}(A(u), B(v)), C(w)),\right. \\
\left.\mathscr{T}\left(A^{\prime}(u), B^{\prime}(v)\right)\right), \quad w \in W
\end{gathered}
$$

where $\mathscr{T}$ is the mapping residual to $\mathscr{I}_{I, t}$.

Proof. From the proof of Theorem 5, we know the mapping $\mathscr{T}$ residual to $\mathscr{I}_{I, t}$ is determined by (12), in which the mapping $T$ on $[0,1]$ is residual to the implication $I$. So $T(1, b)=b$ holds for all $b \in[0,1]$ since $I$ satisfies (P1) (see [23]). (P4) follows that for any $a, b \in[0,1], a=I(1, a) \leqslant I(b, a)$. Further, we get $T(a, b) \leqslant a$ from the residuation condition. Thus, by (12), we have, for any $y \in L_{L^{I}}$,

$$
\begin{aligned}
\mathscr{T}\left(1_{L^{I}}, y\right)= & {\left[T\left(1, y_{1}\right), \max \left\{T\left(1, T\left(y_{2}, t\right)\right), T\left(1, y_{2}\right),\right.\right.} \\
& \left.\left.T\left(1, y_{1}\right)\right\}\right]=\left[y_{1}, \max \left\{T\left(y_{2}, t\right), y_{2}, y_{1}\right\}\right] \\
= & {\left[y_{1}, y_{2}\right]=y . }
\end{aligned}
$$

Therefore, we can obtain (18) by taking $\alpha=1_{L^{I}}$ in (15).

Modus Ponens, i.e., $B$ follows from $A \Rightarrow B$ and $A$, is the most fundamental deduction rule in logic. So, for the reasoning above, it is natural to require that the output $C^{\prime}$ should return to $C$ if $\left(A^{\prime}, B^{\prime}\right)$ is just $(A, B)$. If a reasoning method has this property, we say that it has MP property. Now, We discuss the MP property of triple I reasoning method.

Theorem 9. If the implication I on [0,1] satisfies $(P 1),(P 4),(P 5)$ and $(P)$, then the triple I method given by (18) has MP property for normal inputs, i.e., $A^{\prime}=A$ and $B^{\prime}=B$ imply $C^{\prime}=C$ if $A$ and $B$ are 
normal, i.e., there exist $u_{0} \in U$ and $v_{0} \in V$ such that $A\left(u_{0}\right)=B\left(v_{0}\right)=1_{L^{I}}$

Proof. (P1) follows that the mapping $T$ residual to $I$ satisfies $T(1, b)=b$ for all $b \in[0,1]$. From the proof of Corollary 8 , we know that $I(a, b) \geqslant b$ and $T(a, b) \leqslant a$ for all $a, b \in[0,1]$ since $I$ satisfies (P4). Furthermore, (P4) follows that $T(b, 1)=\inf \{c \in$ $[0,1] \mid b \leqslant I(1, c)\}=\inf \{c \in[0,1] \mid b \leqslant c\}=b$ for any $b \in[0,1]$. So, according to (12) and (8) we get for any $x, y \in L^{I}$,

$$
\begin{aligned}
\mathscr{T}\left(x, 1_{L^{I}}\right)= & {\left[T\left(x_{1}, 1\right), \max \left\{T\left(x_{2}, T(1, t)\right), T\left(x_{1}, 1\right),\right.\right.} \\
& \left.\left.T\left(x_{2}, 1\right)\right\}\right]=\left[x_{1}, \max \left\{T\left(x_{2}, t\right), x_{1}, x_{2}\right\}\right] \\
= & {\left[x_{1}, x_{2}\right]=x, \mathscr{I}_{I, t}\left(1_{L^{I}}, y\right)=\left[\operatorname { m i n } \left\{I\left(1, y_{1}\right),\right.\right.} \\
& \left.\left.I\left(1, y_{2}\right)\right\}, \min \left\{I\left(T(1, t), y_{2}\right), I\left(1, y_{2}\right)\right\}\right] \\
= & {\left[y_{1}, \min \left\{I\left(t, y_{2}\right), y_{2}\right\}\right]=\left[y_{1}, y_{2}\right]=y }
\end{aligned}
$$

Formula (11) follows that the mapping $\mathscr{T}$ is nondecreasing in its first component. From the nonincreasing property of $\mathscr{I}_{I, t}$ with respect to its first component, it follows that $\left\{\gamma \in L^{I} \mid x \leqslant_{L^{I}} \mathscr{I}_{I, t}(z, \gamma)\right\} \subseteq$ $\left\{\gamma \in L^{I} \mid x \leqslant_{L^{I}} \mathscr{I}_{I, t}(y, \gamma)\right\}$ for any $x, y, z \in L^{I}$ and $y \leqslant L_{L^{I}}$ $z$. Hence, for $y \leqslant_{L^{I}} z$ we have $\mathscr{T}(x, y) \leqslant_{L^{I}} \mathscr{T}(x, z)$ for any $x \in L^{I}$. The above description means that $\mathscr{T}$ is nondecreasing in its two components.

Since $A$ and $B$ are normal, there exist $u_{0} \in U$ and $v_{0} \in V$ such that $A\left(u_{0}\right)=B\left(v_{0}\right)=1_{L^{I}}$. Taking $A^{\prime}=A$ and $B^{\prime}=B$ in (18), we have, for any $w \in W$,

$$
\begin{aligned}
C^{\prime}(w)= & \sup _{u \in U, v \in V} \mathscr{T}\left(\mathscr{I}_{I, t}(\mathscr{T}(A(u), B(v)), C(w)),\right. \\
& \mathscr{T}(A(u), B(v))) \geqslant \mathscr{T}\left(\mathscr { I } _ { I , t } \left(\mathscr{T}\left(A\left(u_{0}\right), B\left(v_{0}\right)\right),\right.\right. \\
& \left.C(w)), \mathscr{T}\left(A\left(u_{0}\right), B\left(v_{0}\right)\right)\right) \\
= & \mathscr{T}\left(\mathscr{I}_{I, t}\left(1_{L^{I}}, C(w)\right), 1_{L^{I}}\right)=C(w) .
\end{aligned}
$$

Again, by Theorem 5, (P5) and (P) follows that the residuation condition (10) holds. This implies $\mathscr{T}\left(\mathscr{I}_{I, t}(x, y), x\right) \leqslant_{L^{I}} y$ for any $x, y \in L^{I}$. So we get $C^{\prime}(w) \leqslant L^{I} \sup _{u \in U, v \in V} C(w)=C(w)$ for all $w \in W$.

Summarizing the above, we have $C^{\prime}=C$ if $A^{\prime}=A$ and $B^{\prime}=B$.

In practical applications, we usually use the following distance between two intervals $a=\left[a_{1}, a_{2}\right]$ and $b=\left[b_{1}, b_{2}\right]$ :

$$
d(a, b)=\left|a_{1}-b_{1}\right|+\left|a_{2}-b_{2}\right| .
$$

The uniform metric is commonly used due to its simplicity in computation. So in the sequel, we choose the following uniform metric $d_{U}$ between two IVFSs $A_{1}$ and $A_{2}$ on a universe $W$ :

$$
\begin{gathered}
d_{U}\left(A_{1}, A_{2}\right)=\sup _{w \in W} d\left(A_{1}(w), A_{2}(w)\right)=\sup _{w \in W}\left(\mid \mu_{A_{1}}(w)\right. \\
\left.-\mu_{A_{2}}(w)|+| v_{A_{1}}(w)-v_{A_{2}}(w) \mid\right) .
\end{gathered}
$$

Because most of the practical problems only involve finite elements, and computers can only store finite numbers, in the following discussion in this section we always assume that universes $U, V$ and $W$ are finite.

The following theorem will show that the method (15) is continuous w.r.t. the parameter $\alpha \in L_{I}$, if $T$ in (8) is continuous in its first argument.

Theorem 10. Suppose the conditions in Theorem 6 are satisfied. If $T$ in (8) is continuous in the first argument, then the ouput (15) is continuous w.r.t. the parameter $\alpha$ in the metric $d_{U}$.

Proof. Denote $C^{\prime}$ in $(15)$ as $C_{\alpha}^{\prime}$. We will prove that for any $\alpha^{*} \in L^{I}, \lim _{\alpha \rightarrow \alpha^{*}} d_{U}\left(C_{\alpha}^{\prime}, C_{\alpha^{*}}^{\prime}\right)=0$.

First we prove that if $T$ is continuous in its first argument, then $\mathscr{T}$ is also continuous in its first argument. We only need to prove that for $a, a^{*}, b \in L^{I}$, $\lim _{a \rightarrow a^{*}} d\left(\mathscr{T}(a, b), \mathscr{T}\left(a^{*}, b\right)\right)=0$.

When $a \rightarrow a^{*}$, we have $d\left(a, a^{*}\right)=\mid a_{1}-$ $a_{1}^{*}|+| a_{2}-a_{2}^{*} \mid \rightarrow 0$. So $a_{1} \rightarrow a_{1}^{*}$ and $a_{2} \rightarrow a_{2}^{*}$. Since $T$ is continuous in its first argument, we have $T\left(a_{1}, b_{1}\right) \rightarrow T\left(a_{1}^{*}, b_{1}\right), \quad T\left(a_{2}, T\left(b_{2}, t\right)\right) \rightarrow$ $T\left(a_{2}^{*}, T\left(b_{2}, t\right)\right), \quad T\left(a_{1}, b_{2}\right) \rightarrow T\left(a_{1}^{*}, b_{2}\right) \quad$ and $T\left(a_{2}, b_{1}\right) \rightarrow T\left(a_{2}^{*}, b_{1}\right)$. So

$$
\begin{aligned}
& \max \left\{T\left(a_{2}, T\left(b_{2}, t\right)\right), T\left(a_{1}, b_{2}\right), T\left(a_{2}, b_{1}\right)\right\} \\
& \rightarrow \max \left\{T\left(a_{2}^{*}, T\left(b_{2}, t\right)\right), T\left(a_{1}^{*}, b_{2}\right), T\left(a_{2}^{*}, b_{1}\right)\right\} .
\end{aligned}
$$

Therefore,

$$
\begin{aligned}
& \left|T\left(a_{1}, b_{1}\right)-T\left(a_{1}^{*}, b_{1}\right)\right|+\mid \max \left\{T\left(a_{2}, T\left(b_{2}, t\right)\right),\right. \\
& \left.T\left(a_{1}, b_{2}\right), T\left(a_{2}, b_{1}\right)\right\}-\max \left\{T\left(a_{2}^{*}, T\left(b_{2}, t\right)\right), T\left(a_{1}^{*}, b_{2}\right),\right. \\
& \left.T\left(a_{2}^{*}, b_{1}\right)\right\} \mid \rightarrow 0 .
\end{aligned}
$$

That is, $\lim _{a \rightarrow a^{*}} d\left(\mathscr{T}(a, b), \mathscr{T}\left(a^{*}, b\right)\right)=0$. So $\mathscr{T}$ is continuous in its first argument. 
For all $u \in U, v \in V$ and $w \in W$, we have

$$
\begin{aligned}
& \lim _{\alpha \rightarrow \alpha^{*}} \mathscr{T}\left(\mathscr{T}\left(\alpha, \mathscr{I}_{I, t}(\mathscr{T}(A(u), B(v)), C(w))\right),\right. \\
& \left.\mathscr{T}\left(A^{\prime}(u), B^{\prime}(v)\right)\right) \\
= & \mathscr{T}\left(\lim _{\alpha \rightarrow \alpha^{*}} \mathscr{T}\left(\alpha, \mathscr{I}_{I, t}(\mathscr{T}(A(u), B(v)), C(w))\right),\right. \\
& \left.\mathscr{T}\left(A^{\prime}(u), B^{\prime}(v)\right)\right) \\
= & \mathscr{T}\left(\mathscr{T}\left(\lim _{\alpha \rightarrow \alpha^{*}} \alpha, \mathscr{I}_{I, t}(\mathscr{T}(A(u), B(v)), C(w))\right),\right. \\
& \left.\mathscr{T}\left(A^{\prime}(u), B^{\prime}(v)\right)\right) \\
= & \mathscr{T}\left(\mathscr{T}\left(\alpha^{*}, \mathscr{I}_{I, t}(\mathscr{T}(A(u), B(v)), C(w))\right),\right. \\
& \left.\mathscr{T}\left(A^{\prime}(u), B^{\prime}(v)\right)\right) .
\end{aligned}
$$

So we get

$$
\begin{aligned}
& \lim _{\alpha \rightarrow \alpha^{*}} d\left(\mathscr { T } \left(\mathscr{T}\left(\alpha, \mathscr{I}_{I, t}(\mathscr{T}(A(u), B(v)), C(w))\right),\right.\right. \\
& \left.\mathscr{T}\left(A^{\prime}(u), B^{\prime}(v)\right)\right), \mathscr{T}\left(\mathscr { T } \left(\alpha^{*}, \mathscr{I}_{I, t}(\mathscr{T}(A(u), B(v)),\right.\right. \\
& \left.\left.C(w))), \mathscr{T}\left(A^{\prime}(u), B^{\prime}(v)\right)\right)\right)=0 .
\end{aligned}
$$
have

Since $U, V$ and $W$ contain finite elements, we

$$
\begin{aligned}
& \lim _{\alpha \rightarrow \alpha^{*}} d_{U}\left(C_{\alpha}^{\prime}, C_{\alpha^{*}}^{\prime}\right) \\
= & \lim _{\alpha \rightarrow \alpha^{*}} \sup _{w \in W} d\left(C_{\alpha}^{\prime}(w), C_{\alpha^{*}}^{\prime}(w)\right) \\
= & \lim _{\alpha \rightarrow \alpha^{*}} \sup _{w \in W} d\left(\operatorname { s u p } _ { u \in U , v \in V } \mathscr { T } \left(\mathscr { T } \left(\alpha, \mathscr{I}_{I, t}(\mathscr{T}(A(u), B(v)),\right.\right.\right. \\
& \left.C(w))), \mathscr{T}\left(A^{\prime}(u), B^{\prime}(v)\right)\right), \\
= & \sup _{u \in U, v \in V} \mathscr{T}\left(\mathscr{T}\left(\alpha^{*}, \mathscr{I}_{I, t}(\mathscr{T}(A(u), B(v)), C(w))\right),\right. \\
& \left.\left.\mathscr{T}\left(A^{\prime}(u), B^{\prime}(v)\right)\right)\right) \\
\leqslant & \lim _{\alpha \rightarrow \alpha^{*}} \sup _{w \in W, u \in U, v \in V} d\left(\mathscr { T } \left(\mathscr { T } \left(\alpha, \mathscr{I}_{I, t}(\mathscr{T}(A(u), B(v)),\right.\right.\right. \\
& \left.C(w))), \mathscr{T}\left(A^{\prime}(u), B^{\prime}(v)\right)\right), \mathscr{T}\left(\mathscr { T } \left(\alpha^{*}, \mathscr{I}_{I, t}(\mathscr{T}(A(u),\right.\right. \\
& \left.\left.\left.B(v)), C(w))), \mathscr{T}\left(A^{\prime}(u), B^{\prime}(v)\right)\right)\right)\right) \\
= & \sup _{w \in W, u \in U, v \in V} \lim _{\alpha \rightarrow \alpha^{*}} d\left(\mathscr { T } \left(\mathscr { T } \left(\alpha, \mathscr{I}_{I, t}(\mathscr{T}(A(u), B(v)),\right.\right.\right. \\
& \left.C(w))), \mathscr{T}\left(A^{\prime}(u), B^{\prime}(v)\right)\right), \mathscr{T}\left(\mathscr { T } \left(\alpha^{*}, \mathscr{I}_{I, t}(\mathscr{T}(A(u),\right.\right. \\
& \left.\left.\left.B(v)), C(w))), \mathscr{T}\left(A^{\prime}(u), B^{\prime}(v)\right)\right)\right)\right) \\
= & \sup _{w \in W, u \in U, v \in V} 0=0 .
\end{aligned}
$$

Similarly to Theorem 10, we can prove the following theorem.

Theorem 11. Suppose the conditions in Theorem 6 are satisfied. If $T$ in (8) is continuous, then the output (15) is continuous w.r.t. the inputs $A^{\prime}$ and $B^{\prime}$ in the metric $d_{U}$.

Example 1. Suppose $U=\left\{u_{1}, u_{2}, u_{3}\right\}, \quad V=$ $\left\{v_{1}, v_{2}, v_{3}, v_{4}\right\}, W=\left\{w_{1}, w_{2}, w_{3}\right\}, \alpha=[0.7,0.8]$, $\beta=[0.8,0.9]$,

$$
\begin{aligned}
& A=\frac{[0.3,0.4]}{u_{1}}+\frac{[0.9,1]}{u_{2}}+\frac{[0.7,0.8]}{u_{3}}, \\
& B=\frac{[0.1,0.2]}{v_{1}}+\frac{[0.6,0.7]}{v_{2}}+\frac{[0.9,1]}{v_{3}}+\frac{[0.5,0.6]}{v_{4}}, \\
& C=\frac{[0.6,0.7]}{w_{1}}+\frac{[0.8,0.9]}{w_{2}}+\frac{[0.1,0.2]}{w_{3}}, \\
& A^{\prime}=\frac{[0.4,0.5]}{u_{1}}+\frac{[0.8,1]}{u_{2}}+\frac{[0.8,0.9]}{u_{3}}, \\
& B^{\prime}=\frac{[0.2,0.3]}{v_{1}}+\frac{[0.5,0.7]}{v_{2}}+\frac{[0.8,0.9]}{v_{3}}+\frac{[0.6,0.8]}{v_{4}} .
\end{aligned}
$$

For given rule (13) we calculate $C^{\prime}$ using fully implicational reasoning method (15).

Denote $\quad \mathscr{T}\left(\mathscr{T}\left(\alpha, \mathscr{I}_{I, t}(\mathscr{T}(A(u), B(v)), C(w))\right)\right.$, $\left.\mathscr{T}\left(A^{\prime}(u), B^{\prime}(v)\right)\right)$ as $f(u, v, w)$.

$$
\begin{aligned}
& f\left(u_{1}, v_{1}, w_{1}\right) \\
= & \mathscr{T}\left(\mathscr { T } \left([0.7,0.8], \mathscr{I}_{I, t}(\mathscr{T}([0.3,0.4],[0.1,0.2]),\right.\right. \\
& {[0.6,0.7])), \mathscr{T}([0.4,0.5],[0.2,0.3])) } \\
= & \mathscr{T}\left(\mathscr{T}\left([0.7,0.8], \mathscr{I}_{I, t}([0,0],[0.6,0.7])\right),[0,0]\right) \\
= & \mathscr{T}(\mathscr{T}([0.7,0.8],[1,1]),[0,0]) \\
= & \mathscr{T}([0.7,0.8],[0,0]) \\
= & {[0,0] . }
\end{aligned}
$$

In the same way, we get $f\left(u_{1}, v_{2}, w_{1}\right)=[0,0.4]$, $f\left(u_{1}, v_{3}, w_{1}\right)=[0.4,0.5], \quad f\left(u_{1}, v_{4}, w_{1}\right)=[0,0.5]$, $f\left(u_{2}, v_{1}, w_{1}\right)=[0,0], \quad f\left(u_{2}, v_{2}, w_{1}\right)=[0.5,0.7]$, $f\left(u_{2}, v_{3}, w_{1}\right)=[0.6,0.7], f\left(u_{2}, v_{4}, w_{1}\right)=[0.6,0.7]$, $f\left(u_{3}, v_{1}, w_{1}\right)=[0,0], \quad f\left(u_{3}, v_{2}, w_{1}\right)=[0.5,0.7]$, $f\left(u_{3}, v_{3}, w_{1}\right)=[0.6,0.7], f\left(u_{3}, v_{4}, w_{1}\right)=[0.6,0.7]$. 
So we have

$$
\begin{aligned}
& C^{\prime}\left(w_{1}\right) \\
= & \sup \left\{f\left(u_{1}, v_{1}, w_{1}\right), f\left(u_{1}, v_{2}, w_{1}\right), f\left(u_{1}, v_{3}, w_{1}\right),\right. \\
& f\left(u_{1}, v_{4}, w_{1}\right), f\left(u_{2}, v_{1}, w_{1}\right), f\left(u_{2}, v_{2}, w_{1}\right), \\
& f\left(u_{2}, v_{3}, w_{1}\right), f\left(u_{2}, v_{4}, w_{1}\right), f\left(u_{3}, v_{1}, w_{1}\right), \\
& \left.f\left(u_{3}, v_{2}, w_{1}\right), f\left(u_{3}, v_{3}, w_{1}\right), f\left(u_{3}, v_{4}, w_{1}\right)\right\} \\
= & \sup \{[0,0],[0,0.4],[0.4,0.5],[0,0.5],[0,0],[0.5,0.7], \\
& {[0.6,0.7],[0.6,0.7],[0,0],[0.5,0.7],[0.6,0.7], } \\
& {[0.6,0.7]\}=[0.6,0.7] . }
\end{aligned}
$$

Similarly, we get $C^{\prime}\left(w_{2}\right)=[0.7,0.8], C^{\prime}\left(w_{3}\right)=$ $[0,0.5]$. Therefore,

$$
C^{\prime}=\frac{[0.6,0.7]}{w_{1}}+\frac{[0.7,0.8]}{w_{2}}+\frac{[0,0.5]}{w_{3}} .
$$

\section{Hierarchical full-implicational reasoning method}

Given a fuzzy rule $(A, B) \Rightarrow C$ and an input $\left(A^{\prime}, B^{\prime}\right)$, where $A, A^{\prime}, B, B^{\prime}$ and $C$ are IVFSs on their respective domains $U, V$ and $W$, we now propose hierarchical full-implicational reasoning method as follows:

Step 1) Consider if-then rule $B \Rightarrow C$, then for a given $\beta \in L^{I}$ and the given input $B^{\prime}$, by using the classical $\beta$-triple I method we get the output $C_{1}$ :

$$
\begin{aligned}
& C_{1}(w)=\sup _{v \in V} \mathscr{T}\left(\mathscr{T}\left(\beta, \mathscr{I}_{I, t}(B(v), C(w))\right), B^{\prime}(v)\right), \\
& w \in W
\end{aligned}
$$

Step 2) Consider if-then rule $A \Rightarrow C_{1}$, then for a given $\alpha \in L^{I}$ and the given input $A^{\prime}$, by using $\alpha$-triple I method we get the output $C^{\prime}$ as follows:

$$
\begin{aligned}
C^{\prime}(w)= & \sup _{u \in U} \mathscr{T}\left(\mathscr{T}\left(\alpha, \mathscr{I}_{I, t}\left(A(u), C_{1}(w)\right)\right), A^{\prime}(u)\right) \\
= & \sup _{u \in U} \mathscr{T}\left(\mathscr { T } \left(\alpha, \mathscr{I}_{I, t}\left(A(u), \sup _{v \in V} \mathscr{T}(\mathscr{T}(\beta,\right.\right.\right. \\
& \left.\left.\left.\left.\left.\mathscr{I}_{I, t}(B(v), C(w))\right), B^{\prime}(v)\right)\right)\right), A^{\prime}(u)\right), w \in W
\end{aligned}
$$

where the implication $\mathscr{I}_{I, t}$ on $L^{I}$ is determined by (8) and $\mathscr{T}$ is its residual. We also call the reasoning method determined by (20) hierarchical $(\alpha, \beta)$-triple I method.

Remark 3. In the above hierarchical $(\alpha, \beta)$-triple $I$ method (20), the $\alpha$ is the sustaining degree [13] of $B \Rightarrow C$ to $B^{\prime} \Rightarrow C_{1}$ and the $\beta$ is the one of $A \Rightarrow C_{1}$ to $A^{\prime} \Rightarrow C^{\prime}$, where the definition of sustaining degree of $E \rightarrow F$ to $E^{\prime} \rightarrow F^{\prime}$ is as follows:

$$
\begin{aligned}
& \operatorname{sust}\left(E \rightarrow F, E^{\prime} \rightarrow F^{\prime}\right)=\inf \{(E(u) \rightarrow F(v)) \\
& \left.\rightarrow\left(E^{\prime}(u) \rightarrow F^{\prime}(v)\right) \mid u \in U, v \in V\right\},
\end{aligned}
$$

where $E$ and $F$ are IVFSs on their respective domains $U$ and $V$. The meaning of $\alpha$ and $\beta$ in Section 5 is the same as above.

Especially, if the employed implication $I$ in (8) also satisfies (P1) and (P4), and $\alpha=\beta=1_{L^{I}}$ in (20), then by $\mathscr{T}\left(1_{L^{I}}, y\right)=y$ from the proof of Corollary 8 we have

$$
\begin{aligned}
C^{\prime}(w)= & \sup _{u \in U} \mathscr{T}\left(\mathscr { I } _ { I , t } \left(A(u), \sup _{v \in V} \mathscr{T}\left(\mathscr{I}_{I, t}(B(v), C(w)),\right.\right.\right. \\
& \left.\left.\left.B^{\prime}(v)\right)\right), A^{\prime}(u)\right), w \in W
\end{aligned}
$$

We call the method determined by (21) hierarchical triple I method.

The following theorem shows that the hierarchical triple I method has MP property.

Theorem 12. If the implication I on [0,1] satisfies $(P 1),(P 4),(P 5)$ and $(P)$, then the triple I method given by (21) has MP property for normal inputs, i.e., $A^{\prime}=A$ and $B^{\prime}=B$ imply $C^{\prime}=C$ if $A$ and $B$ are normal.

Proof. From the proof of Theorem 9 we know that $\mathscr{T}\left(x, 1_{L^{I}}\right)=x, \mathscr{I}_{I, t}\left(1_{L^{I}}, y\right)=y$ and $\mathscr{T}\left(\mathscr{I}_{I, t}(x, y), x\right) \leqslant L_{L^{I}} y$ hold for all $x, y \in L^{I}$, and the mapping $\mathscr{T}$ is nondecreasing in its two components. Since $A$ and $B$ are normal, there exist $u_{0} \in U$ and $v_{0} \in V$ such that $A\left(u_{0}\right)=B\left(v_{0}\right)=1_{L^{I}}$. Taking $A^{\prime}=A$ and $B^{\prime}=B$ in (21), we have for any $w \in W$,

$$
\begin{aligned}
C^{\prime}(w)= & \sup _{u \in U} \mathscr{T}\left(\mathscr { I } _ { I , t } \left(A(u), \sup _{v \in V} \mathscr{T}\left(\mathscr{I}_{I, t}(B(v), C(w)),\right.\right.\right. \\
& B(v))), A(u)) \\
\geqslant & \mathscr{T}\left(\mathscr{I}_{I, t}\left(A\left(u_{0}\right), \mathscr{T}\left(\mathscr{I}_{I, t}\left(B\left(v_{0}\right), C(w)\right), B\left(v_{0}\right)\right)\right),\right. \\
& \left.A\left(u_{0}\right)\right)=C(w),
\end{aligned}
$$




$$
\begin{aligned}
& C^{\prime}(w)=\sup _{u \in U} \mathscr{T}\left(\mathscr { I } _ { I , t } \left(A(u), \sup _{v \in V} \mathscr{T}\left(\mathscr{I}_{I, t}(B(v), C(w)),\right.\right.\right. \\
& \quad B(v))), A(u)) \\
& \leqslant L^{I} \sup _{v \in V} \mathscr{T}\left(\mathscr{I}_{I, t}(B(v), C(w)), B(v)\right) \\
& \leqslant{ }_{L^{I}} C(w) .
\end{aligned}
$$

So we get $C^{\prime}(w)=C(w)$ for all $w \in W$, i.e., we obtain that $C^{\prime}=C$ if $A^{\prime}=A$ and $B^{\prime}=B$.

Similarly to Theorem 10 and Theorem 11, we can prove the following two theorems.

Theorem 13. Suppose the conditions in Theorem 3 are satisfied and suppose $U, V$ and $W$ all contain $f$ nite elements. If $T$ in (8) is continuous and I in (8) is continuous in the second argument, then the output (20) is continuous w.r.t. the parameters $\alpha$ and $\beta$ in the metric $d_{U}$.

Theorem 14. Under the same conditions as in Theorem 13, the output (20) is continuous w.r.t. the inputs $A^{\prime}$ and $B^{\prime}$ in the metric $d_{U}$.

If an implication $I$ on $[0,1]$ satisfies (P5) and (P), and its residual mapping $T$ determined by (7) is associative and distributive over the operation max, then it is easy to verify that the mapping $\mathscr{T}$ given by (12), i.e. (11), is also associative. Since the $\mathscr{T}$ and $\mathscr{I}_{I, t}$ defined by (8) are residual to each other and $L^{I}$ is a complete lattice, the associativity of $\mathscr{T}$ is equivalent to the following law of importation:

$$
\mathscr{I}_{I, t}(\mathscr{T}(x, y), z)=\mathscr{I}_{I, t}\left(x, \mathscr{I}_{I, t}(y, z)\right), \quad x, y, z \in L^{I}
$$

Now, we investigate the equivalence between the classical $\alpha$-triple I method (15) and the hierarchical $(\alpha, \beta)$-triple I method (20).

Theorem 15. Assume that the inputs $A^{\prime}$ and $B^{\prime}$ are "singleton" IVFSs on their respective domains $U$ and $V$, then (15) and (20) are equivalent when the implication I on $[0,1]$ satisfies $(P 1),(P 4),(P 5)$ and $(P)$, its residual mapping $T$ determined by (7) is associative and distributive over max, and $\beta=$ $1_{L^{I}}, \alpha \in L^{I}$.

Proof. Assume that the "singleton" IVFSs $A^{\prime}$ and $B^{\prime}$ attain normality at points $u_{0} \in U$ and $v_{0} \in V$, respectively. From the proof of Theorem 9 we know that $\mathscr{T}$ is nondecreasing in its two components, and $\mathscr{T}\left(y, 1_{L^{I}}\right)=\mathscr{T}\left(1_{L^{I}}, y\right)=y$ and $\mathscr{I}_{I, t}\left(1_{L^{I}}, y\right)=y$ hold for all $y \in L^{I}$ under the assumed conditions. It follows from (7) that $T(0, a)=T(a, 0)=0$ for any $a \in[0,1]$ since $I(0, a)=1$ holds. Thus, by an easy calculation we can obtain from (12) that $\mathscr{T}\left(x, 0_{L^{I}}\right)=$ $\mathscr{T}\left(0_{L^{I}}, x\right)=0_{L^{I}}$ for all $x \in L^{I}$. Using the above conditions, we get, for any $w \in W$,

$$
\begin{aligned}
(15)= & C^{\prime}(w) \\
= & \sup _{u \in U, v \in V} \mathscr{T}\left(\mathscr { T } \left(\alpha, \mathscr{I}_{I, t}(\mathscr{T}(A(u), B(v)),\right.\right. \\
& \left.C(w))), \mathscr{T}\left(A^{\prime}(u), B^{\prime}(v)\right)\right) \\
= & \mathscr{T}\left(\mathscr{T}\left(\alpha, \mathscr{I}_{I, t}\left(\mathscr{T}\left(A\left(u_{0}\right), B\left(v_{0}\right)\right), C(w)\right)\right),\right. \\
& \left.\mathscr{T}\left(A^{\prime}\left(u_{0}\right), B^{\prime}\left(v_{0}\right)\right)\right) \\
= & \mathscr{T}\left(\mathscr{T}\left(\alpha, \mathscr{I}_{I, t}\left(\mathscr{T}\left(A\left(u_{0}\right), B\left(v_{0}\right)\right), C(w)\right)\right),\right. \\
& \left.\mathscr{T}\left(1_{L^{I}}, 1_{L^{I}}\right)\right) \\
= & \mathscr{T}\left(\alpha, \mathscr{I}_{I, t}\left(\mathscr{T}\left(A\left(u_{0}\right), B\left(v_{0}\right)\right), C(w)\right)\right), \quad(23)
\end{aligned}
$$

$$
\begin{aligned}
(20)= & C^{\prime}(w) \\
= & \sup _{u \in U} \mathscr{T}\left(\mathscr { T } \left(\alpha, \mathscr{I}_{I, t}\left(A(u), \sup _{v \in V} \mathscr{T}(\mathscr{T}(\beta,\right.\right.\right. \\
& \left.\left.\left.\left.\left.\mathscr{I}_{I, t}(B(v), C(w))\right), B^{\prime}(v)\right)\right)\right), A^{\prime}(u)\right) \\
= & \mathscr{T}\left(\mathscr { T } \left(\alpha, \mathscr{I}_{I, t}\left(A\left(u_{0}\right), \mathscr{T}\left(\mathscr { T } \left(1_{L^{I}},\right.\right.\right.\right.\right. \\
& \left.\left.\left.\left.\left.\mathscr{I}_{I, t}\left(B\left(v_{0}\right), C(w)\right)\right), B^{\prime}\left(v_{0}\right)\right)\right)\right), A^{\prime}\left(u_{0}\right)\right) \\
= & \mathscr{T}\left(\alpha, \mathscr{I}_{I, t}\left(A\left(u_{0}\right), \mathscr{I}_{I, t}\left(B\left(v_{0}\right), C(w)\right)\right)\right)
\end{aligned}
$$

Since $\mathscr{I}_{I, t}$ and $\mathscr{T}$ satisfy the law of importation (22) under the assumed conditions, (23)=(24), i.e., (15) and (20) are equivalent.

It is clear that the conclusion in Theorem 13 is certainly true for the residuum $I$ of any leftcontinuous t-norm on $[0,1]$.

If we restrict our discussion to the ordinary fuzzy sets, then in a similar way as in the above, we can get the hierarchical $(\alpha, \beta)$-triple I method as follows:

$$
\begin{aligned}
C^{\prime}(w)= & \sup _{u \in U} T\left(T \left(\alpha, I\left(A(u), \sup _{v \in V} T(T(\beta, I(B(v),\right.\right.\right. \\
& \left.\left.\left.\left.C(w))), B^{\prime}(v)\right)\right)\right), A^{\prime}(u)\right), w \in W
\end{aligned}
$$

where $I$ is an implication on $[0,1]$ satisfying (P5) and (P), and $T$ is its residual. 
Especially, if $I$ is the residuum of a leftcontinuous t-norm $T, A^{\prime}$ and $B^{\prime}$ are "singleton" fuzzy sets attaining normality at $u_{0} \in U$ and $v_{0} \in V$ respectively, and $\alpha=\beta=1$ in (25), then we have

$$
\begin{aligned}
C^{\prime}(w)= & T\left(T \left(1, I\left(A\left(u_{0}\right), T\left(T\left(1, I\left(B\left(v_{0}\right), C(w)\right)\right),\right.\right.\right.\right. \\
& \left.\left.\left.\left.B^{\prime}\left(v_{0}\right)\right)\right)\right), A^{\prime}\left(u_{0}\right)\right) \\
= & I\left(A\left(u_{0}\right), I\left(B\left(v_{0}\right), C(w)\right)\right) \\
= & I\left(T\left(A\left(u_{0}\right), B\left(v_{0}\right)\right), C(w)\right) .
\end{aligned}
$$

This is the result obtained by CRI or by triple I method or by the hierarchical CRI [3'].

If $I$ in (25) satisfies (P1), then $T(1, b)=b$ for any $b \in[0,1]$. Further, taking $\alpha=\beta=1$ in (25), we have

$$
\begin{gathered}
C^{\prime}(w)=\sup _{u \in U} T\left(I\left(A(u), \sup _{v \in V} T\left(I(B(v), C(w)), B^{\prime}(v)\right)\right),\right. \\
\left.A^{\prime}(u)\right), w \in W
\end{gathered}
$$

If $I$ also satisfies (P2), then $T$ is commutative. Thus, (26) is just the hierarchical CRI given by Balasubramaniam in [34].

In the sequel, we investigate the equivalence between the classical $\alpha$-triple I method (17) and the hierarchical $(\alpha, \beta)$-triple I method (25).

Theorem 16. Assume that the inputs $A^{\prime}$ and $B^{\prime}$ are "singleton" fuzzy sets on their respective domains $U$ and $V$, then (17) and (25) are equivalent when the implication I on $[0,1]$ satisfies $(P 1),(P 4),(P 5)$ and $(P)$, its residual $T$ is associative, and $\alpha \in[0,1], \beta=$ 1.

Proof. Assume that the "singleton" fuzzy sets $A^{\prime}$ and $B^{\prime}$ attain normality at points $u_{0} \in U$ and $v_{0} \in$ $V$, respectively. Since (P1) and (P4) follow that $T(1, b)=T(b, 1)=1$ and $T(a, 0)=T(0, a)=0$ for any $a, b \in[0,1]$, and (P5) and (P) follow that $T$ is nondecreasing in its two components, we get for any $w \in W$,

$$
\begin{aligned}
(17)= & C^{\prime}(w) \\
= & \sup _{u \in U, v \in V} T(T(\alpha, I(T(A(u), B(v)), C(w))), \\
& \left.T\left(A^{\prime}(u), B^{\prime}(v)\right)\right) \\
= & T\left(T\left(\alpha, I\left(T\left(A\left(u_{0}\right), B\left(v_{0}\right)\right), C(w)\right)\right)\right. \\
& \left.T\left(A^{\prime}\left(u_{0}\right), B^{\prime}\left(v_{0}\right)\right)\right) \\
= & T\left(\alpha, I\left(T\left(A\left(u_{0}\right), B\left(v_{0}\right)\right), C(w)\right)\right)
\end{aligned}
$$

$$
\begin{aligned}
(25)= & C^{\prime}(w) \\
= & \sup _{u \in U} T\left(T \left(\alpha, I\left(A(u), \sup _{v \in V} T(T(\beta, I(B(v),\right.\right.\right. \\
& \left.\left.\left.\left.C(w))), B^{\prime}(v)\right)\right)\right), A^{\prime}(u)\right) \\
= & T\left(T \left(\alpha, I\left(A\left(u_{0}\right), T\left(T\left(1, I\left(B\left(v_{0}\right), C(w)\right)\right),\right.\right.\right.\right. \\
& \left.\left.\left.\left.B^{\prime}\left(v_{0}\right)\right)\right)\right), A^{\prime}\left(u_{0}\right)\right) \\
= & T\left(\alpha, I\left(A\left(u_{0}\right), I\left(B\left(v_{0}\right), C(w)\right)\right)\right)
\end{aligned}
$$

Since $I$ and $T$ are residual to each other, the associativity of $T$ is equivalent to $I(T(a, b), c)=$ $I(a, I(b, c))$ for all $a, b, c \in[0,1]$ (see [17]), we obtain that $(27)=(28)$, i.e., (17) and (25) are equivalent.

Example 2. Suppose we have the same $U, V, W, \alpha, \beta, A, B, C, A^{\prime}, B^{\prime}$ and given rule as those in Example 1. Now we calculate $C^{\prime}$ using hierarchical full-implicational reasoning method (20).

$$
\begin{aligned}
C_{1}\left(w_{1}\right)= & \sup \left\{\mathscr { T } \left(\mathscr { T } \left([0.8,0.9], \mathscr{I}_{I, t}([0.1,0.2],\right.\right.\right. \\
& {[0.6,0.7])),[0.2,0.3]), \mathscr{T}(\mathscr{T}([0.8,0.9],} \\
& \left.\left.\mathscr{I}_{I, t}([0.6,0.7],[0.6,0.7])\right),[0.5,0.7]\right), \\
& \mathscr{T}\left(\mathscr{T}\left([0.8,0.9], \mathscr{I}_{I, t}([0.9,1],[0.6,0.7])\right),\right. \\
& {[0.8,0.9]), \mathscr{T}\left(\mathscr { T } \left([0.8,0.9], \mathscr{I}_{I, t}([0.5,0.6],\right.\right.} \\
& {[0.6,0.7])),[0.6,0.8])\} } \\
= & \sup \{[0,0.3],[0.5,0.7],[0.6,0.7],[0.6,0.8]\} \\
= & {[0.6,0.8] . }
\end{aligned}
$$

Then we get

$$
\begin{aligned}
C^{\prime}\left(w_{1}\right)= & \sup \left\{\mathscr { T } \left(\mathscr { T } \left([0.7,0.8], \mathscr{I}_{I, t}([0.3,0.4],\right.\right.\right. \\
& {[0.6,0.8])),[0.4,0.5]) \mathscr{T}(\mathscr{T}([0.7,0.8],} \\
& \left.\left.\mathscr{I}_{I, t}([0.9,1],[0.6,0.8])\right),[0.8,1]\right), \\
& \mathscr{T}\left(\mathscr { T } \left([0.7,0.8], \mathscr{I}_{I, t}([0.7,0.8],[0.6,0.8]),\right.\right. \\
& {[0.8,0.9])\} } \\
= & \sup \{[0.4,0.5],[0.6,0.7],[0.6,0.7]\} \\
= & {[0.6,0.7] . }
\end{aligned}
$$

Similarly, we have $C^{\prime}\left(w_{2}\right)=[0.7,0.8]$ and $C^{\prime}\left(w_{3}\right)=[0.4,0.5]$. Therefore

$$
C^{\prime}=\frac{[0.6,0.7]}{w_{1}}+\frac{[0.7,0.8]}{w_{2}}+\frac{[0.4,0.5]}{w_{3}} .
$$




\section{URC full-implicational reasoning method}

For the interval-valued fuzzy rule (13), i.e. $(A, B) \Rightarrow$ $C$, a given multi-input $\left(A^{\prime}, B^{\prime}\right)$ and $\alpha, \beta \in L^{I}$, to obtain the output $C^{\prime}$, we now propose URC fullimplicational reasoning method as follows:

Step 1) Consider if-then rule $A \Rightarrow C$, then for a given $\alpha \in L^{I}$ and the given input $A^{\prime}$, by $\alpha$-triple I method we get the output $C_{1}^{\prime}$ :

$$
\begin{aligned}
C_{1}^{\prime}(w)= & \sup _{u \in U} \mathscr{T}\left(\mathscr{T}\left(\alpha, \mathscr{I}_{I, t}(A(u), C(w))\right), A^{\prime}(u)\right), \\
& w \in W
\end{aligned}
$$

Step 2) Consider if-then rule $B \Rightarrow C$, then for a given $\beta \in L^{I}$ and the given input $B^{\prime}$, by $\beta$-triple I method we get the output $C_{2}^{\prime}$ as follows:

$$
\begin{aligned}
C_{2}^{\prime}(w)= & \sup _{v \in V} \mathscr{T}\left(\mathscr{T}\left(\beta, \mathscr{I}_{I, t}(B(v), C(w))\right), B^{\prime}(v)\right), \\
& w \in W
\end{aligned}
$$

Taking $C^{\prime}(w)=C_{1}^{\prime}(w) \vee C_{2}^{\prime}(w)$, where $\vee=\max$, we get

$$
\begin{aligned}
C^{\prime}(w)= & \left\{\sup _{u \in U} \mathscr{T}\left(\mathscr{T}\left(\alpha, \mathscr{I}_{I, t}(A(u), C(w))\right), A^{\prime}(u)\right)\right\} \\
& \vee\left\{\sup _{v \in V} \mathscr{T}\left(\mathscr{T}\left(\beta, \mathscr{I}_{I, t}(B(v), C(w))\right), B^{\prime}(v)\right)\right\}
\end{aligned}
$$

where the implication $\mathscr{I}_{I, t}$ on $L^{I}$ is determined by (8) and the $\mathscr{T}$ is its residual. We also call the reasoning method determined by (31) URC $(\alpha, \beta)$-triple I method.

Especially, if the employed implication $I$ satisfies (P1),(P4), (P5) and (P), and $\alpha=\beta=1_{L^{I}}$ in (31), then by $\mathscr{T}\left(1_{L^{I}}, y\right)=y$ from the proof of Corollary 8 , we have for any $w \in W$,

$$
\begin{aligned}
C^{\prime}(w) & =\left\{\sup _{u \in U} \mathscr{T}\left(\mathscr{I}_{I, t}(A(u), C(w)), A^{\prime}(u)\right)\right\} \\
& \vee\left\{\sup _{v \in V} \mathscr{T}\left(\mathscr{I}_{I, t}(B(v), C(w)), B^{\prime}(v)\right)\right\}
\end{aligned}
$$

We call the method determined by (32) URC triple I method.
The following theorem shows that URC triple I method has MP property.

Theorem 17. If the implication I on [0,1] satisfies $(P 1),(P 4),(P 5)$ and $(P)$, then the triple I method given by (32) has MP property for normal inputs, i.e., $A^{\prime}=A$ and $B^{\prime}=B$ imply $C^{\prime}=C$ if $A$ and $B$ are normal.

Proof. From the proof of Theorem 9 we know that $\mathscr{T}\left(x, 1_{L^{I}}\right)=x, \mathscr{I}_{I, t}\left(1_{L^{I}}, y\right)=y$ and $\mathscr{T}\left(\mathscr{I}_{I, t}(x, y), x\right) \leqslant_{L^{I}} y$ hold for all $x, y \in L^{I}$. Since $A$ and $B$ are normal, there exist $u_{0} \in U$ and $v_{0} \in V$ such that $A\left(u_{0}\right)=B\left(v_{0}\right)=1_{L^{I}}$. Taking $A^{\prime}=A$ and $B^{\prime}=B$ in (32), we have for any $w \in W$,

$$
\begin{aligned}
C^{\prime}(w)= & \left\{\sup _{u \in U} \mathscr{T}\left(\mathscr{I}_{I, t}(A(u), C(w)), A(u)\right)\right\} \\
& \vee\left\{\sup _{v \in V} \mathscr{T}\left(\mathscr{I}_{I, t}(B(v), C(w)), B(v)\right)\right\} \\
\geqslant & \mathscr{L ^ { I }}\left(\mathscr{I}_{I, t}\left(A\left(u_{0}\right), C(w)\right), A\left(u_{0}\right)\right) \\
& \vee \mathscr{T}\left(\mathscr{I}_{I, t}\left(B\left(v_{0}\right), C(w)\right), B\left(v_{0}\right)\right) \\
= & C(w) \vee C(w)=C(w), \\
C^{\prime}(w)= & \left\{\sup _{u \in U} \mathscr{T}\left(\mathscr{I}_{I, t}(A(u), C(w)), A(u)\right)\right\} \\
& \vee\left\{\sup _{v \in V} \mathscr{T}\left(\mathscr{I}_{I, t}(B(v), C(w)), B(v)\right)\right\} \\
\leqslant & C(w) \vee C(w)=C(w) .
\end{aligned}
$$

So we get $C^{\prime}(w)=C(w)$ for all $w \in W$, i.e., we obtain that $C^{\prime}=C$ if $A^{\prime}=A$ and $B^{\prime}=B$.

Similarly to Theorem 10 and Theorem 11, we can prove the following two theorems.

Theorem 18. Suppose the conditions in Theorem 3 are satisfied and suppose $U, V$ and $W$ all contain $f$ nite elements. If $T$ in (8) is continuous in the first argument, then the output (31) is continuous w.r.t. the parameters $\alpha$ and $\beta$ in the metric $d_{U}$.

Theorem 19. Suppose the conditions in Theorem 3 are satisfied and suppose $U, V$ and $W$ all contain $f$ nite elements. If $T$ in (8) is continuous in the second argument, then the output (31) is continuous w.r.t. the inputs $A^{\prime}$ and $B^{\prime}$ in the metric $d_{U}$.

Remark 4. The $\alpha$-triple I method (15) and the URC $(\alpha, \beta)$-triple I method (31) are not equivalent in general even if $\alpha=\beta$. In fact, if the inputs 
$A^{\prime}$ and $B^{\prime}$ are "singleton" IVFSs attaining normality at points $u_{0} \in U$ and $v_{0} \in V$ respectively, $\alpha=\beta$, and the employed implication is $\mathscr{I}_{I, t}$ defined by (8), where the implication $I$ on $[0,1]$ satisfies $(\mathrm{P} 1),(\mathrm{P} 4)$, (P5) and (P) and its residual $T$ determined by (7) is distributive over max, then from the proof of Theorem 13 , we have, for any $w \in W$ :

$$
\begin{aligned}
(31)= & C^{\prime}(w) \\
= & \left.\mathscr{T}\left(\mathscr{T}\left(\alpha, \mathscr{I}_{I, t}\left(A\left(u_{0}\right), C(w)\right)\right), A^{\prime}\left(u_{0}\right)\right)\right\} \\
& \vee \mathscr{T}\left(\mathscr{T}\left(\alpha, \mathscr{I}_{I, t}\left(B\left(v_{0}\right), C(w)\right)\right), B^{\prime}\left(v_{0}\right)\right) \\
= & \mathscr{T}\left(\alpha, \mathscr{I}_{I, t}\left(A\left(u_{0}\right), C(w)\right)\right) \\
& \vee \mathscr{T}\left(\alpha, \mathscr{I}_{I, t}\left(B\left(v_{0}\right), C(w)\right)\right)
\end{aligned}
$$

Since the mapping $T$ is distributive over max, i.e. $\vee$, by using the formula (12), it is easy to verify that $\mathscr{T}(\alpha, x \vee y)=\mathscr{T}(\alpha, x) \vee \mathscr{T}(\alpha, y)$ for any $x, y \in L^{I}$. So we get

$$
\begin{aligned}
(31)= & C^{\prime}(w)=\mathscr{T}\left(\alpha, \mathscr{I}_{I, t}\left(A\left(u_{0}\right), C(w)\right)\right. \\
& \left.\vee \mathscr{I}_{I, t}\left(B\left(v_{0}\right), C(w)\right)\right)
\end{aligned}
$$

Comparing (33) with (23), we know (15) is not equivalent to (31) in general since $\mathscr{I}_{I, t}(\mathscr{T}(x, y), z)=$ $\mathscr{I}_{I, t}(x, z) \vee \mathscr{I}_{I, t}(y, z)$ does not hold in general for any $x, y, z \in L^{I}$. Especially, if we take $t=1$, i.e., $\mathscr{T}(x, y)=\left[T\left(x_{1}, y_{1}\right), T\left(x_{2}, y_{2}\right)\right]$, then it is easy to verify that $\mathscr{I}_{I, t}(\mathscr{T}(x, y), z) \geqslant_{L^{I}} \mathscr{I}_{I, t}(x, z) \vee \mathscr{I}_{I, t}(y, z)$ for any $x, y, z \in L^{I}$ and hence we have $(15) \geqslant_{L^{I}}$ (31) in this case. If we restrict our discussion to Zadeh's fuzzy sets, then in a similar way as the above, we can get the URC $(\alpha, \beta)$-triple I method as follows:

$$
\begin{aligned}
C^{\prime}(w)= & \left\{\sup _{u \in U} T\left(T(\alpha, I(A(u), C(w))), A^{\prime}(u)\right)\right\} \vee \\
& \left\{\sup _{v \in V} T\left(T(\beta, I(B(v), C(w))), B^{\prime}(v)\right)\right\}, w \in W
\end{aligned}
$$

where $A, A^{\prime}, B, B^{\prime}, C$ and $C^{\prime}$ are Zadeh fuzzy sets on their respective domains $U, V$ and $W, \alpha, \beta \in[0,1]$, $I$ is an implication on $[0,1]$ satisfying (P5) and (P), and $T$ is the mapping residual to $I$.

Note that if the mapping $T$ in (34) is a t-norm, then it must be left-continuous, this is because that the implication $I$ satisfies (P5) and (P) and hence $T$ and $I$ are residual to each other. Further, if $\alpha=\beta=$
1 , and $A^{\prime}$ and $B^{\prime}$ are "singleton" fuzzy sets attaining normality at $u_{0} \in U$ and $v_{0} \in V$ respectively, then (34) becomes as follows:

$$
\begin{aligned}
C^{\prime}(w)= & T\left(T\left(1, I\left(A\left(u_{0}\right), C(w)\right)\right), A^{\prime}\left(u_{0}\right)\right) \\
& \vee T\left(T\left(1, I\left(B\left(v_{0}\right), C(w)\right)\right), B^{\prime}\left(v_{0}\right)\right) \\
= & I\left(A\left(u_{0}\right), C(w)\right) \vee I\left(B\left(v_{0}\right), C(w)\right) \\
= & I\left(T\left(A\left(u_{0}\right), B\left(v_{0}\right)\right), C(w)\right), \quad w \in W
\end{aligned}
$$

The last step is derived from $I(T(a, b), c)=I(a, c) \vee$ $I(b, c)$ for all $a, b, c \in[0,1]$, which is true for any binary operator $I:[0,1]^{2} \rightarrow[0,1]$ satisfying (P4) (see [47]). (35) is just the result obtained by CRI or by classical triple I method.

About the equivalence between the classical $\alpha$-triple I method (17) and the URC $(\alpha, \beta)$-triple I method (34), we have the following result.

Theorem 20. Assume that the inputs $A^{\prime}$ and $B^{\prime}$ are "singleton" fuzzy sets on their respective domains $U$ and $V$, then (17) and (34) are equivalent when the implication I on $[0,1]$ satisfies (P1),(P4), (P5) and $(P)$, its residual $T$ is distributive over $\vee$, and $\alpha=\beta$.

Proof. Assume that the "singleton" fuzzy sets $A^{\prime}$ and $B^{\prime}$ attain normality at points $u_{0} \in U$ and $v_{0} \in$ $V$, respectively. Since $(\mathrm{P} 1)$ and $(\mathrm{P} 4)$ follow that $T(1, b)=T(b, 1)=1$ and $T(a, 0)=T(0, a)=$ 0 for any $a, b \in[0,1]$, and (P5) and (P) follow that $T$ is nondecreasing in its two components, we get for any $w \in W,(17)=(27)=$ $T\left(\alpha, I\left(T\left(A\left(u_{0}\right), B\left(v_{0}\right)\right), C(w)\right)\right)$, and

$$
\begin{aligned}
(34)= & C^{\prime}(w) \\
= & \left\{\sup _{u \in U} T\left(T(\alpha, I(A(u), C(w))), A^{\prime}(u)\right)\right\} \\
& \vee\left\{\sup _{v \in V} T\left(T(\beta, I(B(v), C(w))), B^{\prime}(v)\right)\right\} \\
= & \left.T\left(T\left(\alpha, I\left(A\left(u_{0}\right), C(w)\right)\right), A^{\prime}\left(u_{0}\right)\right)\right\} \\
& \vee T\left(T\left(\alpha, I\left(B\left(v_{0}\right), C(w)\right)\right), B^{\prime}\left(v_{0}\right)\right) \\
= & T\left(\alpha, I\left(A\left(u_{0}\right), C(w)\right)\right) \vee T\left(\alpha, I\left(B\left(v_{0}\right), C(w)\right)\right) \\
= & T\left(\alpha, I\left(A\left(u_{0}\right), C(w)\right) \vee I\left(B\left(v_{0}\right), C(w)\right)\right)(36)
\end{aligned}
$$

Since (P4) follows that $I(T(a, b), c)=I(a, c) \vee$ $I(b, c)$ holds for any $a, b, c \in[0,1]$, we know $(27)=(36)$, i.e., (17) is equivalent to (34). 
Example 3. Suppose we have the same $U, V, W, \alpha, \beta, A, B, C, A^{\prime}, B^{\prime}$ and given rule as those in Example 1. Now we calculate $C^{\prime}$ using URC full-implicational reasoning method (31).

$$
\begin{aligned}
C_{1}^{\prime}\left(w_{1}\right)= & \sup \left\{\mathscr { T } \left(\mathscr { T } \left([0.7,0.8], \mathscr{I}_{I, t}([0.3,0.4],\right.\right.\right. \\
& {[0.6,0.7])),[0.4,0.5]), \mathscr{T}(\mathscr{T}([0.7,0.8],} \\
& \left.\left.\mathscr{I}_{I, t}([0.9,1],[0.6,0.7])\right),[0.8,1]\right), \\
& \mathscr{T}\left(\mathscr{T}\left([0.7,0.8], \mathscr{I}_{I, t}([0.7,0.8],[0.6,0.7])\right),\right. \\
& {[0.8,0.9])\} } \\
= & \sup \{[0.4,0.5],[0.6,0.7],[0.6,0.7]\} \\
= & {[0.6,0.7] . }
\end{aligned}
$$

$$
\begin{aligned}
C_{2}^{\prime}\left(w_{1}\right)= & \sup \left\{\mathscr { T } \left(\mathscr { T } \left([0.8,0.9], \mathscr{I}_{I, t}([0.1,0.2],\right.\right.\right. \\
& {[0.6,0.7])),[0.2,0.3]), \mathscr{T}(\mathscr{T}([0.8,0.9],} \\
& \left.\left.\mathscr{I}_{I, t}([0.6,0.7],[0.6,0.7])\right),[0.5,0.7]\right) \\
& \mathscr{T}\left(\mathscr{T}\left([0.8,0.9], \mathscr{I}_{I, t}([0.9,1],[0.6,0.7])\right),\right. \\
& {[0.8,0.9]) \mathscr{T}\left(\mathscr { T } \left([0.8,0.9], \mathscr{I}_{I, t}([0.5,0.6],\right.\right.} \\
& {[0.6,0.7])),[0.6,0.8])\} } \\
= & \sup \{[0,0.3],[0.5,0.7]],[0.6,0.7],[0.6,0.8]\} \\
= & {[0.6,0.8] . }
\end{aligned}
$$

$$
\begin{aligned}
C^{\prime}\left(w_{1}\right) & =C_{1}^{\prime}\left(w_{1}\right) \vee C_{2}^{\prime}\left(w_{1}\right)=[0.6,0.7] \vee[0.6,0.8] \\
& =[0.6,0.8] .
\end{aligned}
$$

Similarly, we have $C^{\prime}\left(w_{2}\right)=[0.8,0.8]$ and $C^{\prime}\left(w_{3}\right)=[0,0.5]$. Therefore

$$
C^{\prime}=\frac{[0.6,0.8]}{w_{1}}+\frac{[0.8,0.8]}{w_{2}}+\frac{[0,0.5]}{w_{3}} .
$$

\section{Conclusion}

In this paper, a class of interval-valued fuzzy implications has been constructed, and by means of them, the $\alpha$-triple I and triple I methods for IVFR with multi-antecedent rules have been established. We have also proved that the above triple I method has
MP property is also continuous respectively w.r.t. the parameter $\alpha$ and the inputs. Further, we have applied the fully implicational idea to the hierarchical and URC methods, and proposed hierarchical and URC full-implicational methods which include hierarchical $(\alpha, \beta)$-triple I and triple I methods and URC $(\alpha, \beta)$-triple I and triple I methods. We have proved that the hierarchical and the URC triple I methods have MP property and they are continuous respectively w.r.t. the parameter $(\alpha, \beta)$ and the inputs., the $\alpha$-triple I and the hierarchical $(\alpha, \beta)$-triple I methods are equivalent and the $\alpha$ triple I and the URC $(\alpha, \beta)$-triple I methods are also equivalent under some conditions. Moreover, for the case of Zadeh fuzzy sets, a series of corresponding results have been obtained.

The results obtained in this paper generalized, on the one hand, the fully implicational methods of approximate reasoning from ordinary fuzzy environments to interval-valued fuzzy environments, and on the other hand, proposed new full-implicational methods for fuzzy reasoning. In addition, our computing formulas not only have wide application fields since they cover more implications, but will also bring convenience to analyzing the reasoning methods algebraically and to making further study to their logical foundation.

\section{Acknowledgments}

This work is supported by the National Natural Science Foundation of China (Nos. 61174099, 60774100) and the Natural Science Foundation of Shandong Province of China (No.Y2007A15).

\section{References}

[1] L.A.Zadeh, "Outline of a new approach to the analysis of complex systems and decision processes," IEEE Trans Systems Man and Cybernetics, 3(1), 28-44 (1973).

[2] G.J.Wang, "Fully implicational triple I method for fuzzy reasoning (in Chinese)," Science in China (Series E), 29(1), 43-53 (1999). 
[3] H.W.Liu, G.J.Wang, "Unified forms of fully implicational restriction methods for fuzzy reasoning," Information Sciences, 177(3), 956-966 (2007).

[4] H.W.Liu, G.J.Wang, "A note on the unified forms of triple I method," Computers and Mathematics with Applications, 52(10-11), 1609-1613 (2006).

[5] H.W.Liu, G.J.Wang, "General expressions of triple I restriction methods for fuzzy reasoning," Fuzzy Systems and Mathematics, 22(3), 1-7 (2008).

[6] H.W.Liu, G.J.Wang, "Triple I method based on pointwise sustaining degrees," Computers and Mathematics with Applications, 55, 2680-2688 (2008).

[7] D.W.Pei, "Unified full implication algorithms of fuzzy reasoning," Information Sciences, 178, 520-530 (2008).

[8] D.W.Pei, "On the strict logic foundation of fuzzy reasoning," Soft Computing, 8, 539-545 (2004).

[9] S.Song, C.Feng, E.S.Lee, "Triple I method of fuzzy reasoning," Computer and Mathematics with Applications, 44, 1567-1579 (2002).

[10] S.Song, C.Wu, "Reverse triple I method of fuzzy reasoning,"Since in China (Series F), 45(2), 344-364 (2002).

[11] G.J.Wang, "Triple I method and intervalvalued fuzzy reasoning," Science in China (Series E), 30(4), 331-340 (2000).

[12] X.Y.Wang, "Triple I method of intervalvalued fuzzy reasoning," Appl. Math. J. Chinese Univ. Ser. A (in Chinese), 18(4),467-472 (2003).

[13] G.J.Wang, L.Fu, "Unified forms of triple I method," Computers and Mathematics with Applications, 49, 923-932 (2005).
[14] G.J.Wang, "Formalized theory of general fuzzy reasoning," Information Sciences, 160, 251-266 (2004).

[15] G.J.Wang, "On the logic foundation of fuzzy reasoning," Information Sciences, 117, 47-88 (1999).

[16] G.J.Wang, H.W.Liu, J.S.Song, "A survey of triple I method- its origin, development, application and logical versions (in Chinese)," Fuzzy System and Mathematics, 20(6), 1-14 (2006).

[17] G.J.Wang, "Non-classical Mathematical Logic and Approximate Reasoning (in Chinese)," Science in China Press, Beijing, 2003.

[18] R.Sambuc, Fonctions $\Phi$-floues. "Application à l'aide au diagnostic en pathologie thyroidienne," Ph.D.Thesis, Universite de Marseille, France, 1975.

[19] I.B.Türksen, "Interval-valued fuzzy sets based on normal forms," Fuzzy Sets and Systems, 20, 191-210 (1986).

[20] L.A.Zadeh, "Fuzzy sets," Information and Control, 8(3), 338-356 (1965)

[21] I.B.Türksen, D.D.W.Yao, "Representation of connectives in fuzzy reasoning: The view through normal forms," IEEE Trans. Systems Man Cybernet, 14, 146-151 (1984).

[22] I.B.Türksen, Z.Zhong, "An approximate analogical reasoning schema based on similarity measures and interval-valued fuzzy sets," Fuzzy Sets and Systems, 34, 323-346 (1990).

[23] D.Dzeich, M.B.Gorzalczany, "Application of interval-valued fuzzy sets in signal transmission problems," On Interval and Fuzzy Mathematics, Poland Poznan: Proc Polish Symp 77-82 (1983).

[24] M.B.Gorzałczany, "Approximate inference with interval-valued fuzzy sets-an outline," On Interval and Fuzzy Mathematics, Poland Poznan: Proc Polish Symp 89-95 (1983). 
[25] M.B.Gorzałczany, "A method of inference in approximate reasoning based on intervalvalued fuzzy sets," Fuzzy Sets and Systems, 21, 1-17 (1987).

[26] M.B.Gorzałczany, "Interval-valued fuzzy controller based on verbal model of object," Fuzzy Sets and Systems, 28, 45-53 (1988).

[27] H.Bustince, "Indicator of inclusion grade for interval-valued fuzzy sets. Application to approximate reasoning based on interval-valued fuzzy sets," International Journal of Approximate Reasoning, 23(3), 137-209 (2000).

[28] W.M.Wu, "Principles and Methods of Fuzzy Reasoning," Guizhou Science and Technology Press 1994.

[29] G.J.Wang, X.Y.Wang, J.S.Song, "Refinement of FOOL method for interval-valued fuzzy reasoning," Journal of Shanxi Normal University (Natural Science Edition) (in Chinese), 29(3), 6-19 (2001).

[30] G.Deschrijver, C.Cornelis, E.Kerre, "On the representation of intuitionistic fuzzy t-norms and t-conorms," IEEE Transactions on Fuzzy Systems, 12(1), 45-61 (2004).

[31] G.Deschrijver, C.Cornelis, "Representability in interval-valued fuzzy set theory," International Journal of Uncertainty, Fuzziness and Knowledge-Based Systems, 15(3), 345-361 (2007).

[32] G.Deschrijver, E.E.Kerre, "Implicators based on binary aggregation operators in intervalvalued fuzzy set theory," Fuzzy Sets and Systems, 153, 229-248 (2005).

[33] G.Deschrijver, E.E.Kerre, "Classes of intuitionistic fuzzy t-norms satisfying the residuation principle," International Journal of Uncertainty, Fuzziness and Knowledge-Based Systems, 11(6), 691-709 (2003).

[34] J.Balasubramaniam, "On the law of importation $(x \wedge y) \rightarrow z \equiv(x \rightarrow(y \rightarrow z))$ in fuzzy logic," IEEE Transactions on Fuzzy Systems, 16(1), 130-144 (2008).

[35] W.E.Combs, J.E.Andrews, "Combinatorial rule eliminated by a fuzzy rule configuration," IEEE Transaction on Fuzzy Systems, 6(1), 111 (1998).

[36] M.Baczyński, B.Jayaram, "Fuzzy Implications," Springer, Berlin Heidelberg, 2008.

[37] J.C.Fodor, "A new look at fuzzy connectives," Fuzzy Sets and Systems, 57(2), 141148 (1993).

[38] J.C.Fodor, "Strict preference relations based on weak t-norms," Fuzzy Sets and Systems, 43, 327-336 (1991).

[39] M.Mas,M.Monserrat, J.Torrens, E.Trillas, "A survey on fuzzy implication functions," IEEE Transaction on Fuzzy Systems, 15(6), 11071121 (2007).

[40] J.C.Fodor, "Contrapositive symmetry of fuzzy implications," Fuzzy Sets and Systems, 69, 141-156 (1995).

[41] S.Jenei, "Continuity of left-continuous triangular norms with strong induced negations and their boundary condition," Fuzzy Sets and Systems, 124, 35-41 (2001).

[42] E.P.Klement, M.Navara, "A survey on different triangular norm-based fuzzy logics," Fuzzy Sets and Systems, 101, 241-251 (1999).

[43] G.Deschrijver, E.Kerre, "On the relationship between some extensions of fuzzy set theory," Fuzzy Sets and Systems, 133(2), 227235 (2003).

[44] J.Goguen, "L-fuzzy sets," J. Math. Anal. Appl., 18, 145-174 (1967).

[45] C.Cornelis, G.Deschrijver, E.Kerre, "Implication in intuitionistic fuzzy and intervalvalued fuzzy set theory: construction, classification, application," International Journal of Approximate Reasoning, 35, 55-95 (2004). 
[46] G.Deschrijver, E.E.Kerre, "Smets-Magrez axioms for R-implicators in interval-valued and intuitionistic fuzzy set theory," International Journal of Uncertainty, Fuzziness and Knowledge-Based Systems, 13(4), 453-464
(2005).

[47] J.Balasubramaniam, C,Jagan Mohan Rao, "On the distributivity of importation operators over T and S norms," IEEE Transactions on Fuzzy Systems, 12(2), 194-198 (2004). 